\title{
IGA VASCULITIS: AN UNUSUAL PRESENTATION OF MONONEURITIS MULTIPLEX IN AN ELDERLY WOMAN
}

Marcela Miguens Castelar Pinheiro, ${ }^{1, \star}$, Alexandre Wagner Silva de Souza ${ }^{1}$,Andressa Higa Shinzato ${ }^{1}$, Felipe Lacerda Veiga ${ }^{1}$, Guilherme Pereira Carlesso ${ }^{1}$, Heloísa Rodrigues Silva Catalá ${ }^{1}$, Isadora Medina ${ }^{1}$, Renan Rodrigues Neves Ribeiro do Nascimento ${ }^{1}$, Stella Falcadi Vendramine ${ }^{1}$

1.Universidade Federal de São Paulo, São Paulo (SP), Brazil.

*Corresponding author: marcelacastelar@yahoo.com.br

\section{BACKGROUND}

Immunoglobulin A vasculitis is a systemic small vessel vasculitis that occurs mainly in childhood. The disease is rarer in adults, with an annual incidence of 0.1 to 1.8 per 100,000 inhabitants. The disease is characterized by a tetrad of clinical manifestations: palpable purpura in patients with neither thrombocytopenia nor coagulopathy, arthritis/arthralgia, abdominal pain and renal disease. We selected this case for illustrating two unusual scenarios: an elderly woman with neurological involvement.

\section{CASE REPORT}

A 73-year-old female, with no known comorbidities, came to our service complaining of a purpuric rash on lower limbs (Fig. 1) that started one month earlier. She also presented systemic symptoms, such as daily fever, night sweats, a $5 \mathrm{~kg}$ weight loss, oligoarthralgia and reported bright red blood-stained stools that occurred sporadically during this period. The most disabling symptoms were paresthesia in the lower limbs that quickly evolved to significant neuropathic pain with muscle weakness. Firstly, the main hypothesis at admission was small or medium vessel systemic vasculitis. Then, daily oral prednisone was started at $1 \mathrm{mg} / \mathrm{kg}$ and gabapentin while proceeding with the investigation. Initial lab tests revealed mild anemia, without thrombocytopenia, normal renal function, and urinalysis. Serum tests for infectious diseases were all negative. Imaging studies did not show organomegaly or lymph node enlargement. Electroneuromyography revealed sensory-motor mononeuritis multiplex. Investigations with protein electrophoresis, cryoglobulins and autoantibodies (RF, ANA, anti-dsDNA, anti-ENA, IgM/lgG anticardiolipin, ANCA, anti-MPO, and anti-PR3 antibodies) were negative. Due to the inconclusive findings of a skin biopsy performed at admission and in view of abnormalities in a follow-up urinalysis (WBC: 15 cells/HPF; RBC 85 cells/HPF with dysmorphic cells (++), protein to creatinine ratio: 2.04), a renal biopsy was performed. It revealed diffuse endocapillary glomerulonephritis with glomerular IgA deposits in the direct immunofluorescence, confirming IgAV diagnosis. Due to the severity of the neurological involvement, intravenous pulse therapy with methylprednisolone and cyclophosphamide were added to therapy.

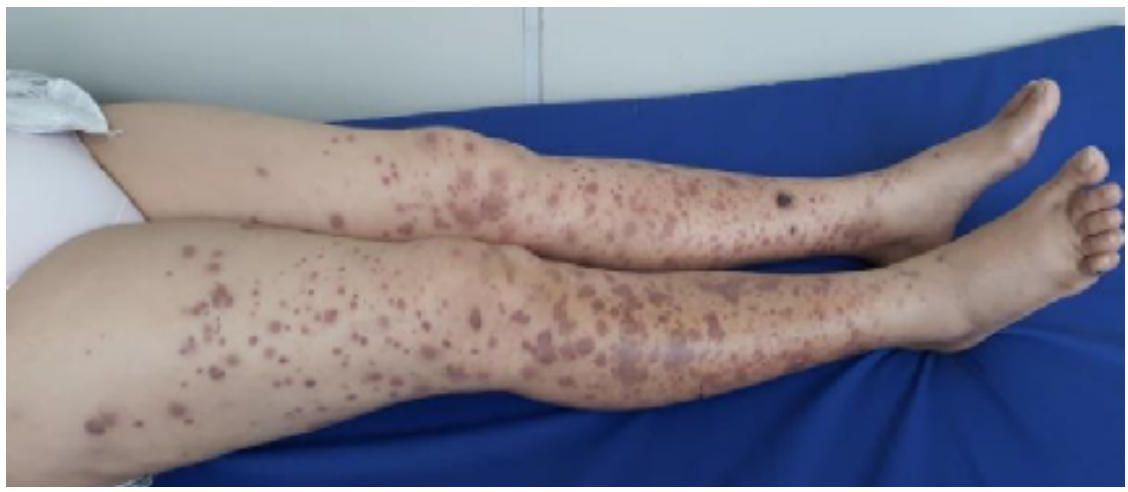

Figure 1. Purpuric rash on lower limbs.

\section{CONCLUSION}

IgAV has a more severe presentation in adults and although neurological manifestations are rare, they often occur in patients with renal impairment and multiorgan involvement. Thereby, we emphasize the importance of including IgAV as a differential diagnosis of mononeuritis multiplex mainly in older age groups with more severe systemic impairment. 